\title{
Convex Sets Strict Separation in Hilbert Spaces
}

\author{
M. A. M. Ferreira ${ }^{1}$ and J. A. Filipe \\ Instituto Universitário de Lisboa (ISCTE - IUL) \\ BRU - IUL, Lisboa, Portugal
}

Copyright (C) 2014 M. A. M. Ferreira and J. A. Filipe. This is an open access article distributed under the Creative Commons Attribution License, which permits unrestricted use, distribution, and reproduction in any medium, provided the original work is properly cited.

\begin{abstract}
Convex sets separation is very important in convex programming, a very powerful mathematical tool for operations research, management and economics, for example. The target of this work is to present Theorem 3.1 that gives sufficient conditions for the strict separation of convex sets.
\end{abstract}

Keywords: Convex sets, strict separation, optimization.

\section{Introduction}

The convex sets separation is very important in convex programming, a very powerful mathematical tool for, see [2,3,4 and 8], operations research, management and economics, namely.

In the mathematical fundamentals of the minimax theorem it is necessary to consider the strict separation of convex sets, see [7]. So the target of this work is to present the Theorem 3.1 that gives sufficient conditions for the strict separation of convex sets.

The results important to establish Theorem 3.1 are outlined in 2 and in 3 that theorem is present. Follow some conclusions and a short list of references.

\section{Supporting Results}

Begin with the following theorem:

\footnotetext{
${ }^{1}$ Corresponding author
} 


\section{Theorem 2.1}

Every closed convex set in a Hilbert space has only one point with minimal norm.

Dem: Call $C$ the closed convex set and $d=\inf \|x\|, x \in C$. Under these conditions it is possible to find a sequence $\left\|x_{n}\right\|$ in $C$, designated minimizing sequence, such that $d=\lim _{n}\left\|x_{n}\right\|$. By the parallelogram law $\left\|\frac{x_{n}-x_{m}}{2}\right\|^{2}=$ $\frac{1}{2}\left(\left\|x_{n}\right\|^{2}+\left\|x_{m}\right\|^{2}\right)-\left\|\frac{1}{2} x_{n}+\frac{1}{2} x_{m}\right\|^{2}$. But, as this equality second member second parcel is a $C$ point square norm, $\left\|\frac{x_{n}-x_{m}}{2}\right\|^{2} \leq \frac{1}{2}\left(\left\|x_{n}\right\|^{2}+\left\|x_{m}\right\|^{2}\right)-$ $d^{2} \rightarrow 0$ and so $x_{n}$ is a Cauchy sequence. As $C$ is closed and every Hilbert space is complete, this limit, call it $z$, belongs to $C$. And, as \|\|$x\|-\| y \| \mid \leq$ $\|x-y\|$, it follows that $\|z\|=d$. Suppose now that $z_{1}$ and $z_{2}$ are two points of $C$ with norm $d$. So, again by the parallelogram law, $\left\|\frac{1}{2}\left(z_{1}-z_{2}\right)\right\|^{2}=d^{2}-$ $\left\|\frac{1}{2}\left(z_{1}+z_{2}\right)\right\|^{2} \leq 0$ and consequently $z_{1}=z_{2}$.

Be now a closed convex set $C$ in a Hilbert space $H$ and a point $x$, anyone, belonging to $H$. As $x-C$ is a closed convex set it results the following Theorem 2.1 corollary:

\section{Corollary 2.1}

Be $C$ a closed convex set in $H$. For any point $x$ of $H$ there is only one point in $C$ that is the closest of $x$; that is: there is one only point $z \in C$ such that $\|x-z\|=\inf \|x-y\|, y \in C$.

So, for the moment, there is an existence and unity result to the optimization problem. But, unhappily, the demonstration is not constructive, not indicating how to determine that unique point. But it is possible to give a better characterization of it, through a variational inequality, as indicated in the next result, which demonstration is centered on a variational argument:

\section{Theorem 2.2}

Be $\quad C$ a closed convex set in $H$. For any point $x$ of $H, z$ is the unique point in $\mathrm{C}$ closest, in norm, from $x$ if and only if

$$
\operatorname{Re}[x-z, y-z] \geq 0, \forall y \in C
$$


Dem: Every characterization of this kind results form a variational argument. So, suppose that $z$ is the only closest point in $C$, guaranteed by Corollary 2.1. So, for every $\theta, 0 \leq \theta \leq 1$, as $C$ is convex, $(1-\theta) z+\theta y \in C$ since $y \in C$. Then

$$
g(\theta)=\|x-((1-\theta) z+\theta y)\|^{2}
$$

is a $\theta$ function twice continuously differentiable. In fact it is a quadratic function of $\theta$. More:

$$
\begin{aligned}
g^{\prime}(\theta)= & 2 \operatorname{Re}[x-\theta y-(1-\theta) z, z-y] \\
& g^{\prime \prime}(\theta)=2 \operatorname{Re}[z-y, z-y] \quad(2.4) .
\end{aligned}
$$

So that $z$ is the minimizing point, it is evident that it must be $g^{\prime}(0) \geq 0 \Leftrightarrow$ $\operatorname{Re}[x-z, z-y] \geq 0$.

Suppose now that (2.1) is satisfied for a certain point $z$ of $C$. Then, constructing again $g(\theta)$ as in (2.2), (2.1) allows to conclude that $g^{\prime}(0)$ is nonnegative and, after (2.4), that $g^{\prime \prime}(\theta)$ is also non-negative. So $g(0) \leq g(1)$ for every $y \in C$, that is $\|x-z\|^{2} \leq\|x-y\|^{2}, \forall y \in C$.

So it was proved that $z$ is the minimizing point in $C$ and, as it was already seen, such point is unique.

\section{Observation:}

-It is interesting to interpret geometrically (2.1).So consider the set of points $\mathrm{h}$ belonging such that $\operatorname{Re}[x-z, h]=c=\operatorname{Re}[x-z, z]$. It is a hiperplane passing by $z$. That hiperplane, which normal is $x-z$ is a support plane from the convex set $C$ in the sense that

$$
\begin{aligned}
& \text { i) } \operatorname{Re}[x-z, z]=c, z \in C \\
& \text { ii) } \operatorname{Re}[x-z, y] \leq c, \forall y \in C
\end{aligned}
$$

since

$\operatorname{Re}[x-z, z-y] \geq 0 \Leftrightarrow \operatorname{Re}[x-z, z]-\operatorname{Re}[x-z, y] \geq 0 \Leftrightarrow \operatorname{Re}[x-z, y] \leq$ $\operatorname{Re}[x-z, z]$. The point $z$ is the supporting point.

Now is the moment to introduce the following definition: 


\section{Definition 2.1}

Given a convex set $C$ in $H$, the map from $H$ to $H$, making correspond to each $x$ the $x$ closest point in $C$, is called projection on $C$ and it is nominated $P_{C}(\cdot) . P_{C}(x)$ is the projection of $x$ over $C$.

And then it follows a Theorem 2.2 corollary:

\section{Corollary 2.2}

If $M$ is a closed vector subspace in $H$, for each $x \in H$ there is one only point of $M$, that is the closest of $x$, being the projection of $x$ on $M$, nominated $P x$, and characterized by

$$
[x-p x, m]=0, \forall m \in M
$$

In this case $P$ is linear and is called projection operator corresponding to $\mathrm{M}$.

\section{The Strict Separation Result}

First the following definitions:

\section{Definition 3.1}

Two closed convex subsets $\mathrm{A}$ and $\mathrm{B}$, in a Hilbert space $H$, are at finite distance from each other if $\inf _{x \in A, y \in B}\|x-y\|=d>0$.

\section{Definition 3.2}

Two closed convex subsets $\mathrm{A}$ and $\mathrm{B}$, in a Hilbert space $H$, are strictly separated if, for some $v \in H, \inf _{x \in A}[v, x]>\sup _{y \in B}[v, y]$.

Then it follows

\section{Theorem 3.1(Strict separation)}

Two closed convex subsets $\mathrm{A}$ and $\mathrm{B}$, in a Hilbert space $H$, at finite distance from each other may be strictly separated.

Dem: In fact, as zero is then a $A-B$ complement interior point, taking its projection over the $A-B$ closure and calling it $v,[-v, v-q] \geq 0, \forall q \in A-$ $B$, by Theorem 2.2. So $[v, q] \geq[v, v]$ and $[v, x]-[v, y] \geq[v, v], x \in A, y \in B$ leading to $\inf _{x \in A}[v, x] \geq \sup _{y \in B}[v, y]$ 
It is also possible to show that

\section{Theorem 3.2}

Being $H$ a finite dimension Hilbert space, if $A$ and $B$ are non-empty disjoint convex sets, they can always be separated.

\section{Conclusions}

As it was established in Theorem 3.1, it is enough that two closed convex sets are at finite distance from each other so that they can be strictly separated in the terms of the Definition 3.2. The conditions are very simple but the demonstration stands on Theorem 2.2 which proof is based on a variational argument.

In finite dimension the situation is much simpler: it is enough that the convex sets, closed or not, are disjoint for they can be separated, as stated in Theorem 3.2.

Finally note that the separation theorems are consequences from the HahnBanach theorem and, when formulated for Hilbert spaces, rephrased applying the Riesz representation theorem, see for instance [6].

\section{References}

[1] A. V. Balakrishnan, Applied Functional Analysis, Springer-Verlag, New York Inc., New York, 1981.

[2] J. von Neuman, O. Morgenstern, Theory of Games and Economic Behavior, John Wiley \& Sons Inc., New York, 1967.

[3] J. P. Aubin, Applied Functional Analysis, John Wiley \& Sons Inc., New York, 1979.

[4] M. A. M. Ferreira, M. Andrade, Management optimization problems, International Journal of Academic Research, 3(2011), 2, Part III, 647-654.

[5] M. A. M. Ferreira, M. Andrade, Separation of a vector space convex parts, International Journal of Academic Research, 4(2012), 2, 5-8.

[6] M. A. M. Ferreira, M. Andrade, The Hahn-Banach theorem and the separation of a vector space convex parts, Journal of Mathematics and Technology, 4(2013), 1, 515. http://www.ijar.lit.az/pdf/jmt/JMT2013\%281-1\%29.pdf 
[7] M. A. M. Ferreira, Convex sets strict separation in the minimax theorem, Applied Mathematical Sciences, 8 (2014), 36, 1781-1787.

http://dx.doi.org/10.12988/ams.2014.4271

[8] M. C. Matos, M. A. M. Ferreira, Game representation-code form, Lecture Notes in Economics and Mathematical Systems, 567, 321-334, 2006. DOI: 10.1007/3-54028727-2_22

Received: April 10, 2014 